\title{
O efeito da divisão de dados na classificação de arritmias usando redes neurais convolucionais
}

\author{
Natália Nagata, Renato Candido e Magno T. M. Silva
}

Resumo-Neste trabalho, avalia-se o efeito da divisão das gravações do sinal de eletrocardiograma de diferentes pacientes entre os conjuntos de treinamento e de teste no diagnóstico automático de arritmias cardíacas. Utilizam-se redes neurais convolucionais para a classificação de batimentos desse sinal. Os resultados indicam que a abordagem que considera os batimentos de um mesmo paciente em ambos os conjuntos leva a métricas de classificação superiores, mas não é clinicamente realista.

Palavras-Chave-Aprendizado de máquina, redes neurais convolucionais, eletrocardiograma, arritmias cardíacas.

Abstract-In this work, we study the effect of dividing the electrocardiogram signal records of different patients between the training and test sets for automatic diagnosis of cardiac arrhythmias. We use convolutional neural network to classify the heartbeats of the signal. The results indicate that using the heartbeats of the same patient in both sets leads to better classification metrics, but is not clinically realistic.

Keywords-Machine learning, convolutional neural networks, electrocardiogram, cardiac arrhythmias.

\section{INTRODUÇÃO}

As arritmias cardíacas são doenças cardiovasculares que se manifestam como uma sequência de batimentos cardíacos de intervalos incomuns [1]. Seu diagnóstico depende de uma análise precisa da atividade elétrica do coração, registrada no sinal de eletrocardiograma (ECG). A análise manual do ECG consome muito tempo dos especialistas e dificulta resultados imediatos [2]. Por isso, um procedimento automatizado pode auxiliar o diagnóstico dessas doenças.

A classificação automática dos batimentos é um problema complexo, uma vez que as características morfológicas e temporais do sinal dependem do paciente e de sua condição física [3]. Como o sinal varia entre os pacientes, é possível que pacientes diferentes apresentem sinais de ECG com morfologias distintas para a mesma doença e que doenças diferentes alterem os sinais de ECG aproximadamente da mesma maneira [3]. Ainda hoje, o diagnóstico de arritmias cardíacas é realizado por cardiologistas, dadas as altas taxas de erro de interpretação por técnicas computadorizadas [4].

Em geral, o diagnóstico de arritmias é dividido em quatro etapas: o pré-processamento do sinal, a segmentação, a extração das características e a classificação. Em trabalhos recentes, redes neurais convolucionais (convolutional neural network - CNN) foram usadas para aprender características úteis do sinal de ECG de forma automática, eliminando a necessidade da etapa de extração de características. Entretanto, em muitos

Natália Nagata, Renato Candido, Magno T. M. Silva, Depto. de Engenharia de Sistemas Eletrônicos, Escola Politécnica, Universidade de São Paulo, São Paulo, SP, Brasil, e-mails: nagata.natalia@usp.br; renatocan@lps.usp.br; magno.silva@usp.br. Este trabalho foi financiado pela FAPESP (2019/26911-6 e 2021/02063-6) e CAPES (código de financiamento 001). desses trabalhos, os autores não seguem as recomendações da Association for the Advancement of Medical Instrumentation (AAMI) [5] e utilizam batimentos de um mesmo paciente tanto no conjunto de treinamento, quanto no de teste. Com exceção de métodos direcionados para o monitoramento de pacientes específicos [6], essa abordagem não é clinicamente realista, pois as características do sinal de ECG são distintas para cada indivíduo e as redes podem ser favorecidas pelo aprendizado das particularidades de cada um.

Neste artigo, estuda-se o impacto de se utilizar os batimentos de um mesmo paciente nas fases de treinamento e de teste de redes neurais convolucionais para o diagnóstico de arritmias cardíacas. Foram utilizadas as gravações do banco de dados MIT-BIH Arrhythmia Database (MITDB) [7]. Os resultados de [1], obtidos considerando-se os batimentos de um mesmo paciente nas duas fases, foram reproduzidos e a mesma arquitetura foi utilizada para a abordagem em que os dados de um mesmo paciente são utilizados em apenas uma das fases. Dessa forma, foi possível comparar os resultados e avaliar o efeito da divisão dos dados.

\section{FORMULAÇ̃̃O DO PROBLEMA}

Para reproduzir os resultados de [1], foram utilizadas 260 amostras da primeira derivação do sinal de ECG em torno de um complexo QRS central como entrada da rede. Seguindo as recomendações da AAMI, as anotações dos batimentos foram agrupadas em cinco classes: batimentos do nó sinoatrial $(\mathrm{N})$, supraventriculares ectópicos $(\mathrm{S})$, ventriculares ectópicos $(\mathrm{V})$, fusão de batimentos normais e ventriculares ectópicos (F) e desconhecidos ou de marca-passo (Q). Utilizou-se a entrada do conjunto A de [1], normalizando-se os dados por $(\mathbf{x}-\mu) / \sigma$, em que $\mathrm{x}$ é um exemplo de treinamento, $\mu$ é a sua média e $\sigma$ é o seu desvio padrão.

As duas abordagens consideradas para a divisão dos dados são: (i) usar os batimentos dos mesmos pacientes nas duas fases da rede, dividindo-se os dados aleatoriamente em 10 conjuntos com a mesma quantidade de batimentos e realizando uma validação cruzada ( $k$-fold cross validation), em que 9 conjuntos são usados para o treinamento da rede e 1 é usado para o teste; e (ii) separar os dados de cada fase considerando gravações inteiras dos pacientes, sem repetir pacientes entre as fases, seguindo-se a divisão proposta por [2] e excluindose os pacientes com marca-passos (sinais 102, 104, 107 e 217) como recomendado pela AAMI. Na abordagem (i), o número de dados da classe Q é consideravelmente maior do que na segunda, devido à exclusão desses pacientes.

Foram utilizadas as bibliotecas Tensorflow e Keras [8] para as simulações. A CNN reproduzida de [1] possui 3 camadas convolucionais, 3 camadas com funções Max-Pooling e 3 
camadas totalmente conectadas. Na ordem de propagação, as camadas convolucionais possuem números de filtros (kernel) iguais a 5,10 e 20 e tamanhos dos filtros iguais a 3, 4 e 4. Todos os filtros das camadas de pooling possuem tamanho igual a 2 e deslocam-se com um passo (stride) igual a 2. As camadas totalmente conectadas possuem, na ordem de propagação, 30, 20 e 5 neurônios. Foram usadas funções de ativação LeakyReLU $\operatorname{com} \alpha=0,1$ nas camadas convolucionais e nas camadas totalmente conectadas, além da função Softmax na camada de saída. A arquitetura reproduzida neste trabalho difere da implementada por [1] nas funções de ativação e na regularização: o valor de $\alpha$ não foi especificado em [1] e a regularização $L_{2} \operatorname{com} \lambda=0,2$ usada em [1] não levou a bons resultados aqui e por isso não foi considerada. Considerouse ainda o gradiente descendente com momento $(\beta=0,7)$, o passo de aprendizado de $\eta=0,003,20$ épocas para o treinamento, mini-batches de tamanho $k=10$, inicialização de Xavier para os pesos e a função custo de entropia cruzada categórica. A arquitetura dessa rede está ilustrada na Figura 1.

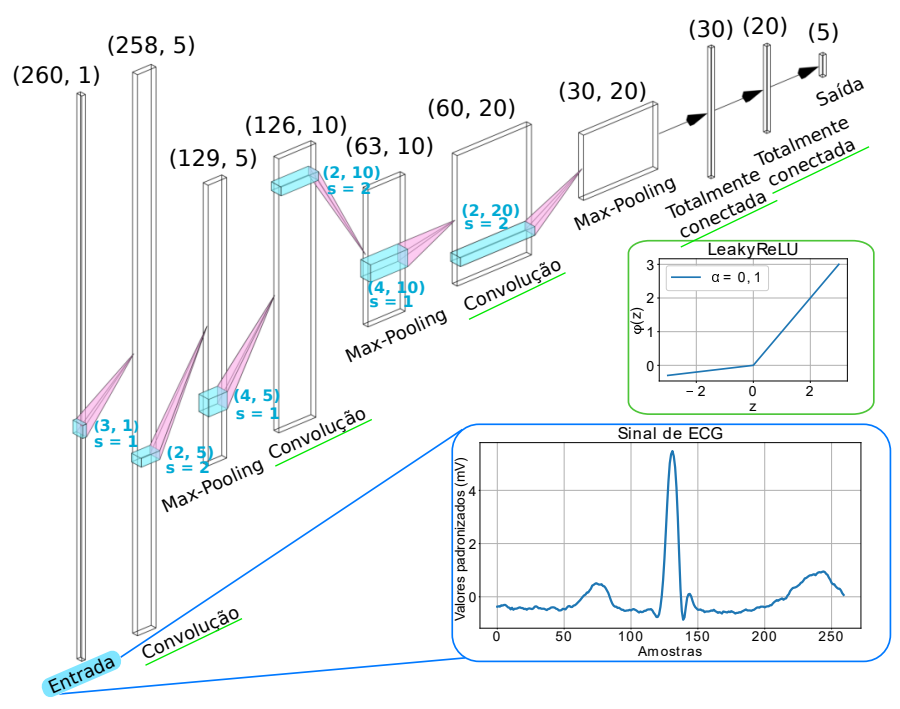

Fig. 1: Arquitetura da CNN.

\section{Resultados E Discuss Ão}

Para cada abordagem, calcularam-se as métricas de Acurácia geral $A c c=(V P+V N) /(V P+V N+F P+F N)$, de Sensibilidade $S e=V P /(V P+F N)$ e de Precisão $P=$ $V P /(V P+F P)$ para cada classe, sendo $V P, V N, F P$ e $F N$ as quantidades de verdadeiros positivos, verdadeiros negativos, falsos positivos e falsos negativos, respectivamente. Seguindo a recomendação da AAMI, os falsos positivos devido à classe $\mathrm{F}$ foram desconsiderados para a classe $\mathrm{V}$ e os falsos positivos devido à classe $\mathrm{Q}$ foram desconsiderados para a classe $\mathrm{S}$.

A Tabela I apresenta as métricas de [1] e as obtidas para as duas abordagens. Também são apresentados os resultados de [2], considerado como estado da arte na abordagem de divisão realista dos dados. Em [2], utiliza-se a análise de discriminantes lineares para classificar as arritmias a partir de características extraídas do ECG em uma etapa anterior.

Os resultados obtidos com a CNN reproduzida neste trabalho para a abordagem (i) são próximos dos relatados por [1] e apresentam valores acima de $70 \%$ de $A c c$, Se e $P$ para todas as classes. A rede apresentou ainda valores de $P$ das
TABELA I: Métricas (\%) dos resultados das simulações.

\begin{tabular}{|c|c|c|c|c|c|c|c|c|c|c|c|}
\hline \multirow{2}{*}{ Abordagem } & \multirow{2}{*}{ Acc } & \multicolumn{2}{|c|}{$\mathrm{N}$} & \multicolumn{2}{|c|}{$S$} & \multicolumn{2}{|c|}{$\mathrm{V}$} & \multicolumn{2}{|c|}{$F$} & \multicolumn{2}{|c|}{$\mathrm{Q}$} \\
\hline & & $\mathrm{Se}$ & $\bar{P}$ & $\mathrm{Se}$ & $\bar{P}$ & $\mathrm{Se}$ & $\mathrm{P}$ & $\mathrm{Se}$ & $\mathrm{P}$ & Se & $\mathrm{P}$ \\
\hline CNN reproduzida & 98,8 & 99,6 & 99,2 & 83,5 & 92,4 & 96,9 & 97,5 & 74,3 & 90,2 & 98 , & 99,6 \\
\hline (1) Acharya et al. [1] & 89,1 & 88,4 & 99,0 & 85,3 & 31,4 & 92,7 & 78,6 & 88,2 & 17,8 & 95,5 & 90,9 \\
\hline CNN reproduzida & 88,2 & 93,2 & 93,8 & 2,9 & 9,0 & 79,6 & 66,7 & 0,0 & 0,0 & 14,3 & 0,0 \\
\hline Chazal et al. [2] & 85,9 & 86,9 & 99,2 & 75,9 & 38,5 & 77,7 & 81,9 & 89,4 & 8,6 & 0 & 0 \\
\hline
\end{tabular}

classes S, V e F maiores do que os de [1]. No entanto, ao se considerar a arquitetura idêntica para a abordagem (ii), os acertos da rede diminuem significativamente e são obtidas métricas muito baixas para as classes $\mathrm{S}, \mathrm{F}$ e Q. Isso se deve à menor quantidade de dados dessas classes, que prejudica o desempenho da rede, principalmente quando não são utilizadas ponderações de acordo com essas quantidades, como funções custo com pesos para lidar com o desbalanceamento.

Em [1], também são apresentados resultados de classificação com todas as métricas maiores do que $90 \%$, considerando a abordagem (i) com exemplos sintéticos. É evidente que sobreamostrar os exemplos na abordagem (i), usando o desvio padrão e a média dos exemplos originais, favorece a rede com o overfitting provocado pela repetição dos dados. No entanto, esses resultados não são realistas, uma vez que na prática, o classificador será usado para classificar o ECG de um paciente que não foi usado no treinamento. Por fim, cabe observar que a extração de características automática das CNNs não favorece a classificação na abordagem (ii), o que pode ser comprovado comparando seus resultados com os de [2].

\section{Conclusões}

Diversos pesquisadores têm trabalhado para melhorar as técnicas computadorizadas de identificação de arritmias cardíacas. No entanto, muitos trabalhos otimizam classificadores para resultados que não são realistas. Neste trabalho, estudou-se o efeito do uso de diferentes abordagens nas CNNs. Verificou-se que os resultados podem ser facilmente favorecidos ao se considerar os batimentos de um determinado paciente como independentes. Observou-se ainda que o desbalanceamento dos dados prejudica a obtenção de bons classificadores na abordagem realista e a sobreamostragem melhora o desempenho para uma abordagem não realista, sendo recomendado usar pesos nas funções custo na primeira.

\section{REFERENCIAS}

[1] U. R. Acharya et al. "A deep convolutional neural network model to classify heartbeats," Comput. Biol. Med., vol. 89, pp. 389-396, 2017.

[2] P. de Chazal, M. O'Dwyer, and R. B. Reilly, "Automatic classification of heartbeats using ECG morphology and heartbeat interval features," IEEE Trans. Biomed. Eng., vol. 51, pp. 1196-1206, 2004.

[3] S. K. Berkaya et al., "A survey on ECG analysis," Biomedical Signal Processing and Control, vol. 43, pp. 216-235, 2018.

[4] A. P. Shah and S. A. Rubin, "Errors in the computerized electrocardiogram interpretation of cardiac rhythm," Journal of Electrocardiology, vol. 40, pp. 385-390, 2007.

[5] Association for the Advancement of Medical Instrumentation (AAMI), "ANSI/AAMI EC57:1998/(R)2008 - Testing and reporting performance results of cardiac rhythm and ST segment measurement algorithms," American National Standards Institute, Arlington, VA, USA, 2008.

[6] S. Kiranyaz, T. Ince, and M. Gabbouj, "Real-time patient-specific ECG classification by 1-D convolutional neural networks," IEEE Trans. Biomed. Eng., vol. 63, pp. 664-675, 2015.

[7] A. L. Goldberger et al., "PhysioBank, PhysioToolkit, and PhysioNet: Components of a new research resource for complex physiologic signals," Circulation, vol. 101, pp. e215-e220, Jun. 13, 2000.

[8] Martín Abadi et al., "TensorFlow: Large-scale machine learning on heterogeneous systems," 2015, Software available from tensorflow.org. 